\title{
Motion Model Selection in Tracking Humans
}

\author{
Damien Kelly ${ }^{\dagger}$ and Frank Boland* \\ Department of Electronic and Electrical Engineering \\ Trinity College Dublin \\ IRELAND \\ E-mail: ${ }^{\dagger}$ kelly16@tcd.ie ${ }^{*}$ fboland@tcd.ie
}

\begin{abstract}
The performance of many human tracking algorithms rely on accurate motion models. Due to the nature of human motion it is often difficult to determine the suitability of a chosen model. It is typically the case that over the tracking duration the characteristics of the observed motion will fit many different models. Commonly used motion models in the area of tracking include the constant position (CP), constant velocity (CV) and constant acceleration (CA) motion models. This paper applies the Kalman filtering algorithm to the problem of tracking a person's position using a finite set of different motion models. The statistical properties of the innovation sequence are used as a basis for motion model selection.

Keywords - Kalman Filter, Motion Modelling, Tracking
\end{abstract}

\section{INTRODUCTION}

The successful determination of a moving person's position requires the filtering of useful information from state observations made in the presence of noise. In order to extract this information it is necessary to make assumptions on the characteristic motion of the person being tracked. Although this may be a simple assumption such as for example, the person existing in $2 \mathrm{D}$ space, it may also be an assumption on the nature of their underlying movements. In general the more precise a priori knowledge of the person's characteristic motion that can be determined, the more useful information can be extracted from noisy position measurements.

It is often the case in object tracking algorithms that a priori knowledge is incorporated into the estimation procedure through the assumption of a motion model. Many motion models have been proposed in the area of tracking such as, the constant position, constant acceleration and constant velocity models [1]. Although these motion models have found applications in many areas of tracking, it is often difficult to assign an object's motion to one particular model. It may be sufficient, for example, to assume constant acceleration when tracking the trajectory of a falling object, although this assumption could not be justifiably applied to tracking motion of a changeable nature such as that of human motion. Typically, in moving, a human will transgress over many different mod- els. For this reason in tracking humans it is often difficult to determine a suitable model. One common model used in people tracking which reflects unpredictability is that of the Brownian motion model. This particular model assumes independent direction of motion at each time step. It does not however reflect the intuition that often over long durations human motion is correlated in time.

One technique in tracking which addresses the problem of changing dynamics is the use of adaptive motion models which enable the target's motion characteristics to change appropriately over time [2]. The problem with this approach is in defining the criteria by which the motion model should be adapted. This requires continuous monitoring of the tracking filter's performance whereby any deviation from it's expected performance would indicate a degradation in the accuracy of the position estimates.

This paper examines motion model selection as applied to the problem of tracking human movements through the use of the Kalman filter algorithm. The statistical properties of the innovation sequence of the Kalman filter are examined in detecting divergence in the Kalman filter resulting from modelling inaccuracies. The remaining sections of this paper are organised as follows. Section II gives an overview of kinematic state estimation. Section III examines a simulated tracking problem. In Section IV, motion model selection in a real tracking problem is examined. Section V 
presents discussion and conclusions.

\section{BACKGROUND}

A common approach in tracking is to approximate the position $x_{k}$ of a target by it's Taylor series expansion [2] i.e.,

$x_{k+1}=x_{k}+T \dot{x}_{k}+\frac{T^{2}}{2} \ddot{x}_{k}+\ldots$

where the time interval $k$ to $k+1$ is of duration $T$. It is well known that a correct approximation requires all derivatives of the position. Where such information is not possible to determine a truncated Taylor series is used. This is equivalent to assuming the target trajectory to follow some polynomial of finite order. In order to relax this assumption the highest order derivative of the truncated Taylor series is often modelled as a random process. The derivatives of position define the moving target's kinematic state model $\mathbf{x}_{\mathbf{k}}$ i.e. $\mathbf{x}_{k}=\left[x_{k} \dot{x}_{k} \ldots{ }^{n} x_{k}^{-1}\right]^{\prime}$ where $x_{k}$ denotes the position and the kinematic state model is of dimension $n$. The primary aim in tracking is to estimate the evolution of the state over time.

\section{a) Kinematic State Estimation}

The estimated kinematic state process with control input $\tilde{\mathbf{u}}_{k}$ and measurement process $\tilde{\mathbf{z}}_{k}$ required to implement the Kalman tracking filter are,

$\tilde{\mathbf{x}}_{k+1}=\tilde{\mathbf{A}}_{k} \tilde{\mathbf{x}}_{k}+\tilde{\mathbf{B}}_{k} \tilde{\mathbf{u}}_{k}+\tilde{\mathbf{C}}_{k} \tilde{v}_{k}$

$\tilde{\mathbf{z}}_{k+1}=\tilde{\mathbf{H}}_{k+1} \tilde{\mathbf{x}}_{k+1}+\tilde{\mathbf{G}}_{k+1} \tilde{w}_{k+1}$

where $\tilde{\mathbf{H}}_{k+1}$ is the measurement matrix and $\tilde{\mathbf{B}}_{k}$, $\tilde{\mathbf{C}}_{k}$ and $\tilde{\mathbf{G}}_{k}$ denote the gain matrices corresponding to the control input $\tilde{\mathbf{u}}_{k}$, process noise $\tilde{v}_{k}$ and measurement noise $\tilde{w}_{k}$ respectively. The process noise $\tilde{v}_{k}$ and measurement noise $\tilde{w}_{k}$ are assumed to be zero mean, independent Gaussian random processes with associated covariance matrices,

$\delta_{k l} \tilde{\mathbf{Q}}_{k}=E\left[\tilde{\mathbf{C}}_{k} \tilde{v}_{k} \tilde{v}_{l}^{\prime} \tilde{\mathbf{C}}_{l}^{\prime}\right]$,

$\delta_{k l} \tilde{\mathbf{R}}_{k}=E\left[\tilde{\mathbf{G}}_{k} \tilde{w}_{k} \tilde{w}_{l}^{\prime} \tilde{\mathbf{G}}_{l}^{\prime}\right]$

where $\delta_{k l}$ denotes the kronecker delta function.

Using the state model of (2) the equations which describe the discrete Kalman filter are

$$
\begin{aligned}
& \hat{\tilde{\mathbf{x}}}_{k+1 \mid k+1}=\hat{\tilde{\mathbf{x}}}_{k+1 \mid k}+\tilde{\mathbf{K}}_{k+1} \tilde{\nu}_{k+1} \\
& \tilde{\nu}_{k+1}=\tilde{\mathbf{z}}_{k+1}-\tilde{\mathbf{H}}_{k+1} \hat{\tilde{\mathbf{x}}}_{k+1 \mid k} \\
& \hat{\tilde{\mathbf{x}}}_{k+1 \mid k}=\tilde{\mathbf{A}}_{k} \hat{\tilde{\mathbf{x}}}_{k \mid k} \\
& \tilde{\mathbf{P}}_{k+1 \mid k}=\tilde{\mathbf{A}}_{k+1} \tilde{\mathbf{P}}_{k \mid k} \tilde{\mathbf{A}}_{k+1}^{\prime}+\tilde{\mathbf{Q}}_{k+1}
\end{aligned}
$$

$$
\begin{aligned}
& \tilde{\mathbf{P}}_{k+1 \mid k+1}=\left(\mathbf{I}-\tilde{\mathbf{K}}_{k+1} \tilde{\mathbf{H}}_{k+1}\right) \tilde{\mathbf{P}}_{k+1 \mid k} \\
& \tilde{\mathbf{S}}_{k+1}=\tilde{\mathbf{H}}_{k+1} \tilde{\mathbf{P}}_{k+1 \mid k} \tilde{\mathbf{H}}_{k+1}^{\prime}+\tilde{\mathbf{R}}_{k+1} \\
& \tilde{\mathbf{K}}_{k+1}=\tilde{\mathbf{P}}_{k+1 \mid k} \tilde{\mathbf{H}}_{k+1}^{\prime} \tilde{\mathbf{S}}_{k+1}^{-1} .
\end{aligned}
$$

where $\hat{\tilde{\mathbf{x}}}$ is filtered state estimate, $\tilde{\mathbf{P}}$ is the covariance of the error in the state estimate, $\tilde{\mathbf{K}}$ is the Kalman gain, $\tilde{\nu}$ is the innovation sequence and $\tilde{\mathbf{S}}$ is the covariance of the innovation. The notations $k \mid k-1$ and $k \mid k$ are used to denote the prediction and filtering steps of the Kalman filter respectively. The state transition and noise gain matrices of typical motion models and the models referred to in this paper are shown in Table 1 for reference.

\begin{tabular}{|c|c|c|}
\hline Model & $\tilde{\mathbf{A}}_{k}$ & $\tilde{\mathbf{C}}_{k}$ \\
\hline \hline $\mathrm{CP}$ & {$[1]$} & {$[T]$} \\
\hline $\mathrm{CV}$ & {$\left[\begin{array}{ll}1 & T \\
0 & 1\end{array}\right]$} & {$\left[\begin{array}{c}\frac{T}{2}^{2} \\
T\end{array}\right]$} \\
\hline $\mathrm{CA}$ & {$\left[\begin{array}{ccc}1 & T & \frac{T}{2}^{2} \\
0 & 1 & T \\
0 & 0 & 1\end{array}\right]$} & {$\left[\begin{array}{c}\frac{T}{6}^{3} \\
\frac{T}{2}^{2} \\
T\end{array}\right]$} \\
\hline
\end{tabular}

Table. 1: Transition and process noise gain matrices for a constant position model CP, constant velocity model $\mathrm{CV}$ and constant acceleration model CA.

Where the state model of (2) represents the true state model, the Kalman gain is optimal and the error in the state estimate is minimum in a mean square sense. In general this is not the case and the state model of (2) represents only an estimate of some true state model,

$$
\begin{aligned}
& \mathbf{x}_{k+1}=\mathbf{A}_{k} \mathbf{x}_{k}+\mathbf{B}_{k} \mathbf{u}_{k}+\mathbf{C}_{k} v_{k} \\
& \mathbf{z}_{k+1}=\mathbf{H}_{k} \mathbf{x}_{k+1}+\mathbf{G}_{k+1} w_{k+1} .
\end{aligned}
$$

In the remainder of this discussion the estimated observation matrix $\tilde{\mathbf{H}}_{k}$ is taken to be $\tilde{\mathbf{H}}_{k}=\mathbf{H}_{k}$ i.e. the estimated observation matrix is know to be correct.

\section{b) Divergence in the Kalman Filter}

Divergence in the Kalman filter occurs when the error in the filtered state estimate does not correspond to that determined by the Kalman filter equations (4) - (10). Divergence in the Kalman filter can be classified as either true divergence, where the errors become unbounded, or apparent divergence where finite degradations are observed 
in the filtered state estimates [3]. This paper is concerned with apparent divergence in the tracking Kalman filter due to inaccuracies in motion modelling.

It can be seen from equation (5) that the innovation sequence represents the error in the predicted measurement based on model (2). For this reason it does not represent the true innovation. It is clear that the innovation in relation to the true state model of (11) can be obtained by replacing $\tilde{\mathbf{z}}_{k+1}$ in equation (5) by $\mathbf{z}_{k+1}$. The actual innovation is therefore [4],

$\nu_{k+1}=\mathbf{z}_{k+1}-\tilde{\mathbf{H}}_{k+1} \hat{\tilde{\mathbf{x}}}_{k+1 \mid k}$

Substituting this expression for the true innovation into equation (4), the true error in the filtered state estimate,

$\mathbf{e}_{k+1 \mid k+1}=\mathbf{x}_{k+1}-\hat{\tilde{\mathbf{x}}}_{k+1 \mid k+1}$,

after some manipulation is found to be,

$$
\begin{aligned}
\mathbf{e}_{k+1 \mid k+1}= & \phi_{k+1} \tilde{\mathbf{A}}_{k} \mathbf{e}_{k \mid k}+\phi_{k+1} \tilde{\mathbf{B}}_{k}\left(\mathbf{u}_{k}-\tilde{\mathbf{u}}_{k}\right) \\
& +\phi_{k+1} \Delta \tilde{\mathbf{A}}_{k} \mathbf{x}_{k}+\phi_{k+1} \Delta \tilde{\mathbf{B}}_{k} \mathbf{u}_{k} \\
& +\phi_{k+1} \mathbf{C}_{k} v_{k}-\tilde{\mathbf{K}}_{k+1} \mathbf{G}_{k+1} w_{k}
\end{aligned}
$$

where $\phi_{k+1}=\left[\mathbf{I}-\tilde{\mathbf{K}}_{k+1} \mathbf{H}_{k+1}\right], \Delta \tilde{\mathbf{A}}_{k}=\mathbf{A}_{k}-\tilde{\mathbf{A}}_{k}$ and $\Delta \tilde{\mathbf{B}}_{k}=\mathbf{B}_{k}-\tilde{\mathbf{B}}_{k}$.

In the case where the motion model of (2) represents the true motion, the innovation sequence can be shown to be zero mean and orthogonal [5]. In expanding equation (12), it can be seen that all modelling errors manifest in the innovation sequence i.e.,

$$
\begin{aligned}
\nu_{k+1}= & \mathbf{H}_{k+1} \tilde{\mathbf{A}}_{k} \mathbf{e}_{k \mid k}+\mathbf{H}_{k+1} \Delta \tilde{\mathbf{A}}_{k} \mathbf{x}_{k} \\
& +\mathbf{H}_{k+1} \tilde{\mathbf{B}}_{k}\left(\mathbf{u}_{k}-\tilde{\mathbf{u}}_{k}\right) \\
& +\mathbf{H}_{k+1} \Delta \tilde{\mathbf{B}}_{k} \mathbf{u}_{k}+\mathbf{H}_{k+1} \mathbf{C}_{k} v_{k} \\
& +\mathbf{G}_{k+1} w_{k+1} .
\end{aligned}
$$

It is clear that continually monitoring the statistical properties of the innovation sequence provides a basis for detecting modelling errors. This is the basis of many existing methods of divergence detection and control in the Kalman filter [6]. Of particular interest in the area of motion model selection from a finite set of models, is the problem of determining the most appropriate model from the observed divergence. The following section examines a finite set of motion models and innovation sequences of the corresponding tracking filters in a computer simulated tracking problem.

\section{Computer Simulated Tracking Problem}

The class of motion models examined in this section is defined in Table 2. This table defines a set of motion models for the tracking scenarios of a person not moving (CP), moving at constant velocity (CV) and moving with constant acceleration (CA). Also shown in the table is the actual simulated model (Act.). This model represents a controllable system whereby all states are reachable through acceleration or deceleration. The controllable acceleration component corresponds to the entry $a$ in matrix $\mathbf{u}_{k}$.

In relation to equation (16), it can be seen that if $E\left[\mathbf{w}_{k}\right]$ is zero mean then the error due to an inaccurate motion model propagates in the expected value of the innovation sequence through $\Delta \tilde{\mathbf{A}}_{k}$, $\Delta \tilde{\mathbf{B}}_{k}, \tilde{\mathbf{B}}_{k}$ and $v_{k}$. In this simulated case, $E\left[v_{k}\right]$ is the change in acceleration over the time step $T$. Over periods of constant acceleration therefore, $E\left[v_{k}\right]=0$. Taking $\tilde{\mathbf{u}}_{k}=0$ from Table 2, the expected value of the innovation sequence in the simulated case becomes,

$$
\begin{aligned}
E\left[\nu_{k+1}\right]= & \mathbf{H}_{k+1} \tilde{\mathbf{A}}_{k} E\left[\mathbf{e}_{k \mid k}\right]+ \\
& +\mathbf{H}_{k+1} \Delta \tilde{\mathbf{A}}_{k} \mathbf{x}_{k}+\mathbf{H}_{k+1} \tilde{\mathbf{B}}_{k} \mathbf{u}_{k} \\
& +\mathbf{H}_{k+1} \Delta \tilde{\mathbf{B}}_{k} \mathbf{u}_{k}+\mathbf{H}_{k+1} \mathbf{C}_{k} E\left[v_{k}\right] \\
= & \mathbf{H}_{k+1}\left[\tilde{\mathbf{A}}_{k} E\left[\mathbf{e}_{k \mid k}\right]+\Delta \tilde{\mathbf{A}}_{k} \mathbf{x}_{k}+\mathbf{B}_{k} \mathbf{u}_{k}\right. \\
& \left.+\mathbf{C}_{k} E\left[v_{k}\right]\right]
\end{aligned}
$$

The following examines $E\left[\nu_{k}\right]$ in tracking the simulated motion using the $\mathrm{CP}, \mathrm{CV}$ and $\mathrm{CA}$ models.

- Using the CP Motion Model

$$
\Delta \tilde{\mathbf{A}}_{k}=\left[\begin{array}{ccc}
0 & T & \frac{T}{2}^{2} \\
0 & 1 & T \\
0 & 0 & 1
\end{array}\right] \mathbf{B}_{k} \mathbf{u}_{k}=\left[\begin{array}{c}
0 \\
a \\
0
\end{array}\right]
$$

$E\left[\nu_{k+1}\right]=E\left[\mathbf{e}_{k \mid k}\right]+T \dot{x}_{k}+\frac{T^{2}}{2} \ddot{x}_{k}+\frac{T^{3}}{6} E\left[v_{k}\right]$

- Using the CV Motion Model

$$
\Delta \tilde{\mathbf{A}}_{k}=\left[\begin{array}{ccc}
0 & 0 & \frac{T}{2}^{2} \\
0 & 0 & T \\
0 & 0 & 1
\end{array}\right] \mathbf{B}_{k} \mathbf{u}_{k}=\left[\begin{array}{c}
0 \\
a \\
0
\end{array}\right]
$$

$E\left[\nu_{k+1}\right]=E\left[\mathbf{e}_{k \mid k}\right]+\frac{T^{2}}{2} \ddot{x}_{k}+\frac{T}{6}^{3} E\left[v_{k}\right]$

- Using the CA Motion Model

$$
\Delta \tilde{\mathbf{A}}_{k}=\left[\begin{array}{ccc}
0 & 0 & 0 \\
0 & 0 & 0 \\
0 & 0 & 0
\end{array}\right] \mathbf{B}_{k} \mathbf{u}_{k}=\left[\begin{array}{l}
0 \\
a \\
0
\end{array}\right]
$$

$E\left[\nu_{k+1}\right]=E\left[\mathbf{e}_{k \mid k}\right]+\frac{T^{3}}{6} E\left[v_{k}\right]$ 


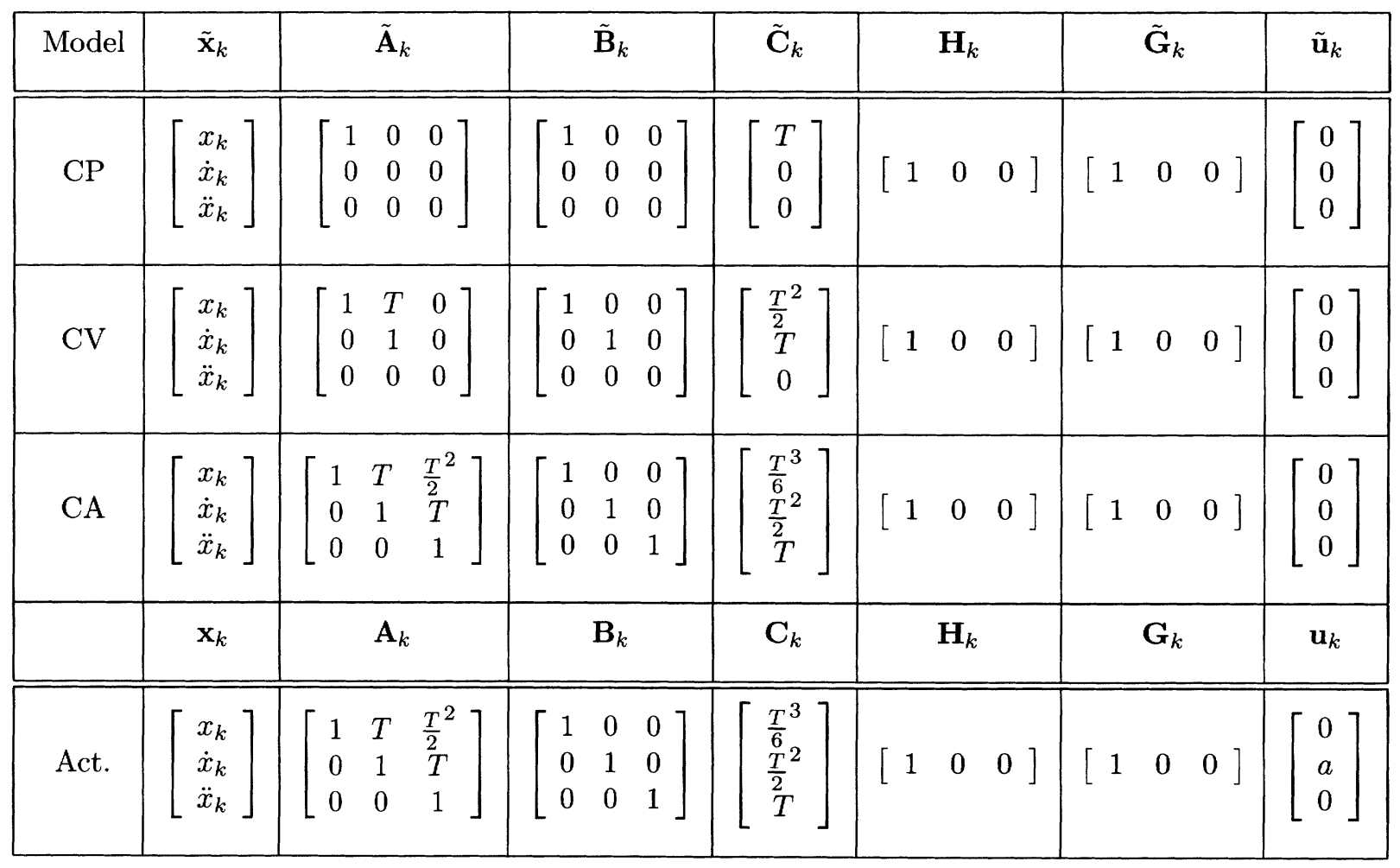

Table. 2: Matrices for a constant position model CP, constant velocity model CV and constant acceleration model CA.

Summarised in Table 3 is the expected value of the innovation sequence $E\left[\nu_{k}\right]$ using the $\mathrm{CP}, \mathrm{CV}$ and CA models, under the simulated scenarios of a person not moving $\left(\dot{x}_{k}=0\right)$, moving with constant velocity $\left(\dot{x}_{k} \neq 0\right)$ and moving with constant acceleration.

From Table 3 it can be seen that in using a CP motion model over periods of constant acceleration $\left(\ddot{x}_{k}=a\right)$, the expected value of the innovation sequence increases linearly. Using a CV motion model results in an offset in the expected value of the innovation sequence. As the CA motion model estimates all states of the simulated motion it is the optimal model and is therefore zero mean. Similarly, over periods of constant velocity both the CV and CA motion models are zero mean and an offset is observed in $E\left[\nu_{k}\right]$ where the CP model is used. This is illustrated in fig. 1, where the simulated tracking problem is that of a person starting from an initial stationary position to a state of constant velocity and then decelerating back to a stationary position.

\section{Motion Model Selection in a Real Tracking APPLICATION}

The motion models of Table 2 were applied to the problem of tracking a person in an image sequence. The image sequence used, consisted of a person walking on a grid ${ }^{1}$ of known width $(4.35 \mathrm{~m})$ and length $(5 \mathrm{~m})$. The video tracking method of background subtraction was used to locate the person in each frame. The captured background was taken from a section of the image sequence where the grid was unoccupied. The use of background subtraction and the application of a suitable threshold to account for changes in lighting, enabled the person to be located in each frame of the image sequence. The person's position on the grid was determined through a mapping of frame co-ordinates to grid co-ordinates. The mapping of frame co-ordinates to positions on the grid was achieved using a technique which exploits the fact that the intersection of the diagonals of a trapezoid are invariant under perspective distortion [7]. This recursive algorithm firstly determines the person's y position on the grid in a similar manner to the secant method for root finding. Secondly the person's $\mathrm{x}$ position is determined in relation to the grid boundaries. Knowing the grid dimensions and also the location of the corners of the grid therefore enabled the geometry of the frame to be mapped to that of the geometry of the grid. An example frame from the image sequence used is shown in fig. 2. In this sequence the person walks horizontally across the full width of the grid.

Shown in fig. 3 (a) are the 1D position measurements $\mathbf{z}_{k}$, of the person for each of the 188 frames of the image sequence. Due to the frame resolution of $576 \times 720$ and the area occupied by the grid within the frame, 


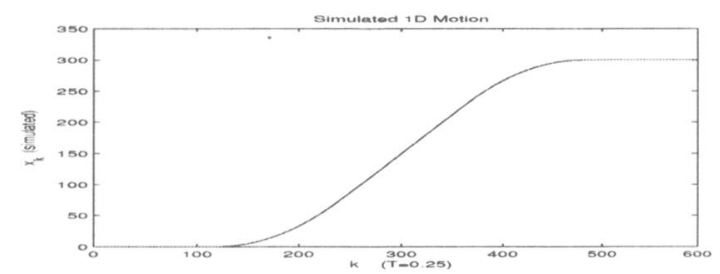

(a)

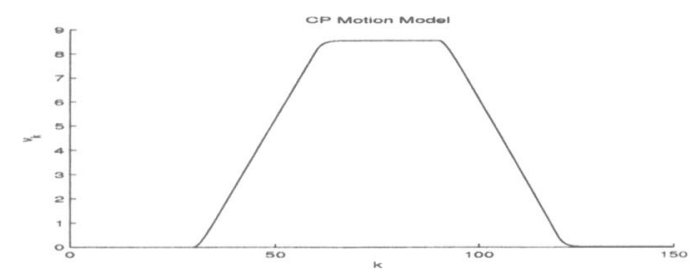

(b)

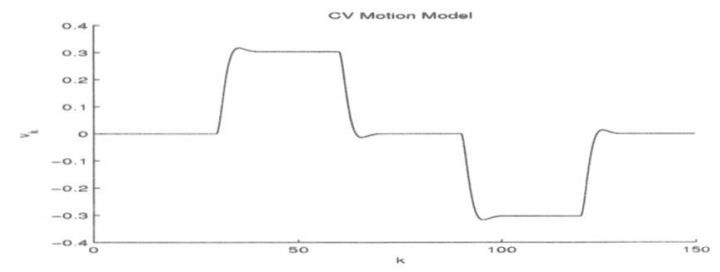

(c)

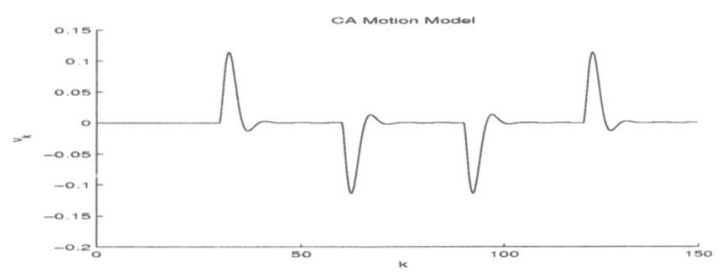

(d)

Fig. 1: Innovation sequences for the CP (a), CV (b) and $\mathrm{CA}$ (c) motion models in a computer simulated tracking problem. The simulated data is that of a person starting at an initial stationary position at $k=0$ and accelerating over $k=30$ to $k=60$, maintaining a constant velocity $(5 \mathrm{~m} / \mathrm{s})$ from $k=60$ to $k=90$ and then decelerating back to a stationary position at $k=120$. $(T=0.25 s)$.

\begin{tabular}{|c||c||c|}
\hline \multicolumn{1}{|c||}{} & \multicolumn{2}{c|}{ Motion Scenario } \\
\cline { 2 - 3 } & $\mathrm{CV}$ & $\mathrm{CA}$ \\
\hline \hline $\mathrm{CP}$ & $E\left[\mathbf{e}_{k \mid k}\right]+T \dot{x}_{k}$ & $E\left[\mathbf{e}_{k \mid k}\right]+T \dot{x}_{k}+\frac{T}{2}^{2} \ddot{x}_{k}$ \\
\hline $\mathrm{CV}$ & $E\left[\mathbf{e}_{k \mid k}\right]$ & $E\left[\mathbf{e}_{k \mid k}\right]+\frac{T^{2}}{2} \ddot{x}_{k}$ \\
\hline $\mathrm{CA}$ & $E\left[\mathbf{e}_{k \mid k}\right]$ & $E\left[\mathbf{e}_{k \mid k}\right]$ \\
\hline
\end{tabular}

Table. 3: Expected value of the true innovation $\nu_{k+1}$ for different estimating models under motion scenarios of constant acceleration (CA) and constant velocity (CV). the position measurements were determined to be accurate to $0.01 \mathrm{~m}$ over the mid section of the grid. For this reason the variance of the measurement noise $w_{k}$ was chosen to be $\sigma_{w}^{2}=0.0001 \mathrm{~m}^{2}$. In examining the position measurements, suitable values for the process noise $\sigma_{v}^{2}$ were determined to be $\sigma_{v}^{2}=0.04 m^{2}, \sigma_{v}^{2}=0.25 \frac{m^{2}}{s^{2}}$ and $\sigma_{v}^{2}=0.25 \frac{m^{2}}{s^{4}}$ for the $\mathrm{CP}, \mathrm{CV}$ and $\mathrm{CA}$ Motion Models respectively. Similarly in the case of the computer simulated tracking problem the control input was assumed to be $\tilde{\mathbf{u}}_{k}=0$. The innovation sequences of the Kalman filters applied to the tracking problem using these three motion models are shown in fig. 3 .

As can be seen in fig. 3 (b) the offset in the mean of the innovation sequence corresponding to the CP model indicates the person is moving with constant velocity. The suitability of a CV motion model is seen in fig. 3 (c) where after the initial divergence of the filter, the innovation sequence is zero mean over the period of constant velocity. It is also seen in fig. 3 (d) that the higher dimension CA model also results in a zero mean innovation sequence but time required for the filter to converge after the initial divergence is greater.

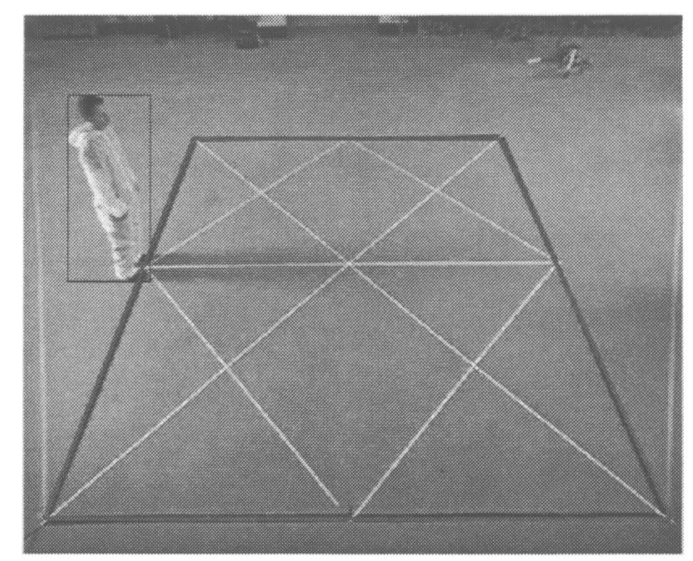

Fig. 2: Frame from the image sequence of a person walking across a grid. The video tracking technique of background subtraction was used to determine a bounding box for the person in each frame.

\section{Discussion and Conclusions}

The successful tracking of humans using the Kalman filter requires accurate motion models. Due to the nature of human motion it is difficult to determine the suitability of a particular model. Inaccurate motion modelling causes bounded divergence in the Kalman filter resulting in biased position estimates. In order to correct the estimates obtained from the filter it is necessary to control the divergence by adapting or changing the model. 


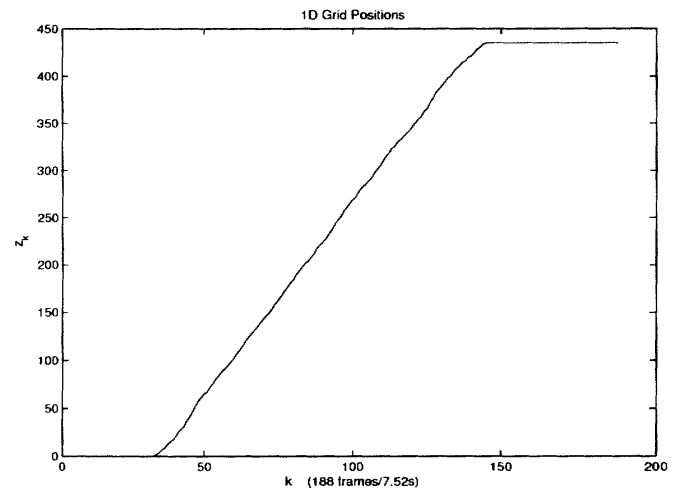

(a)

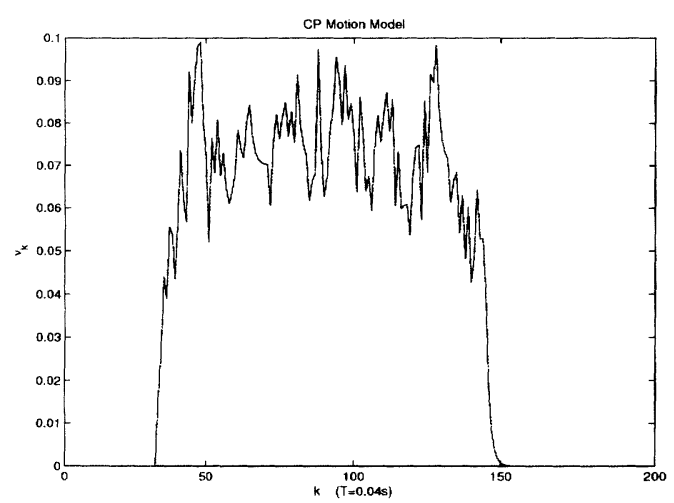

(b)

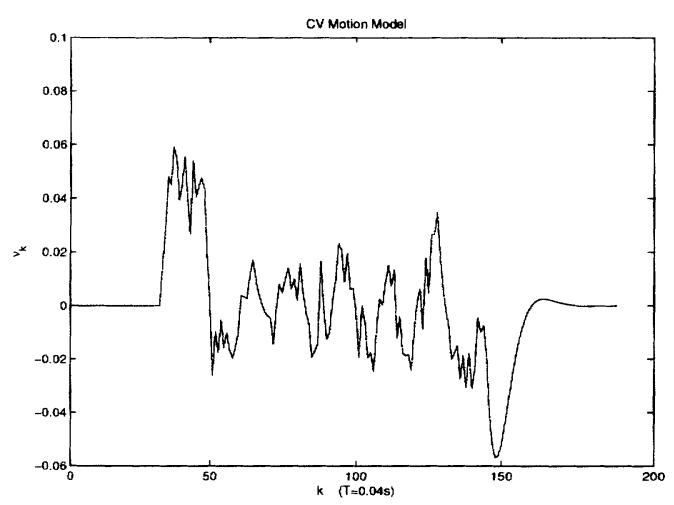

(c)

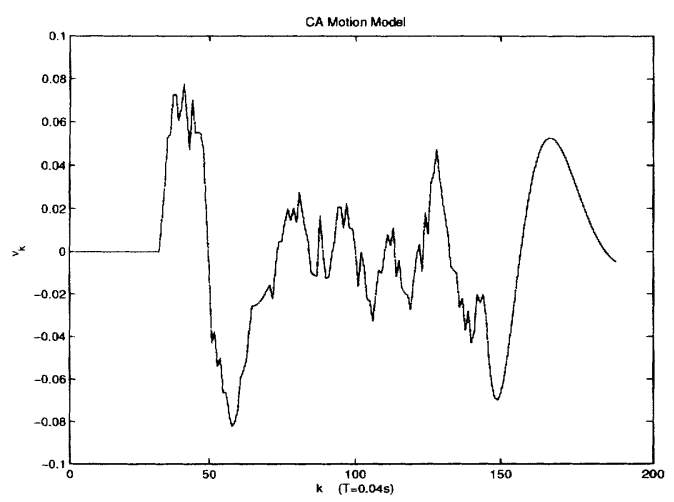

(d)

Fig. 3: Innovation sequences of the Kalman filter for the CP (a), CV (b) and CA (c) motion models as applied to the problem of tracking the person in the image sequence. $(T=0.04 s)$
It has been shown that the tracking performance of a particular motion model can be determined through monitoring of the innovation sequence. The performance of a finite set of motion models was examined under various simulated motion scenarios. It was seen that the deviation in the expected value of the innovation sequence provided a basis for not only detecting divergence in the filter but also for classifying the motion being tracked.

The particular set of motion models used enabled the observed motion to be classified as either fitting a $\mathrm{CP}, \mathrm{CV}$ or CA model. In this way monitoring the innovation sequence was seen to provide a basis for motion model selection.

\section{ACKNOWLEDGEMENTS}

Damien Kelly gratefully acknowledges the support of the Irish Research Council for Science, Engineering and Technology (IRCSET), through an Embark Initiative research scholarship.

\section{REFERENCES}

[1] X. Rong Li, Vesselin P. Jilkov. Survey of Maneuvering Target Tracking. Part 1: Dynamic Models. IEEE Trans. on Aerospace and Electronic Systems, 39:1333-1364, 2003.

[2] M. Moore, J. Wang. Adaptive Dynamic Modelling for Kinematic Positioning. Probabilistic and Non-Probabilistic Assessment in Data Analysis, IAG Scientific Asembly, Budapest, Hungary, paper 4, 2001.

[3] Robert J. Fitzgerald. Divergence of the Kalman Filter. IEEE Trans. on Automatic Control, 16:736-747, 1971.

[4] Charles F. Price. An Analysis of the Divergence Problem in the Kalman Filter. IEEE Trans. on Automatic Control, 13:699-702, 1968.

[5] Y. Bar-Shalom, X. Rong Li, T. Kirubarajan. Estimation with Applications to Tracking and Navigation. Wiley-Interscience, 2001.

[6] F. M. Boland, H. Nicholson. Control of Divergence in Kalman Filters. Electronics Letters, 12:367-369, 1976.

[7] H. Denman, N. Rea, A. Kokaram. Contentbased Analysis for Video from Snooker Broadcasts. Journal of Computer Vision and Image Understanding, 92:176-195, 2003.

\footnotetext{
${ }^{1}$ Audio and video data of people walking and talking on a grid was collected for investigations in tracking people using audio and video. An online database of the collected data is currently being compiled.
} 